\title{
Tierra natal: entre agonía y afirmación de la diferencia 1
}

\section{(Homeland: between agony and assertion of the difference)}

\author{
Alfredo ROCHA DE LA TORRE
}

Recibido: 29 de enero de 2012

Aceptado: 2 de abril de 2012

\section{Resumen}

Este artículo aborda la 'tierra natal' como expresión de la pregunta heideggeriana por 'el sentido de lo que es'. Técnica y civilización mundial son consideradas aquí como tendencias hacia la homogenización de la experiencia humana y como pérdida de 'lo propio' representado en la tierra natal, lo que conduce a la disolución de la diferencia y de la interpelación ética del Otro. La filosofía de Heidegger, por el contrario, permite afirmar la tierra natal como apertura a la interpelación del 'otro'.

Palabras clave: tierra natal, técnica, civilización mundial, ontología, ética y diferencia.

\section{Abstract}

This article tackles the concept of "homeland" as expression of the Heideggerian question concerning 'the meaning of being'. Technology and global civilisation are considered here as tendencies to the homogenisation of the human experience and as loss of "the own-ness' of the homeland, what guides to the dissolution of the difference and of the ethical appeal of the other. On the contrary, Heideggers philosophy allows to affirm the homeland as disclosure to the appeal of the 'other'.

Keywords: Homeland, technology, global civilisation, ontology, ethics, difference.

\footnotetext{
1 El presente trabajo es un producto de mi investigación como Gastwissenschaftler en la Bergische Universität Wuppertal, Alemania, "Der Andere im Denken Martin Heideggers: Eine Antwort auf die Kritik von Emmanuel Levinas", apoyado por la Alexander von Humboldt-Stiftung.
} 
Las reflexiones de Martin Heidegger acerca de la técnica y el pensamiento que calcula (das rechnende Denken), que conducen finalmente hacia el fenómeno de la civilización mundial (Weltzivilisation) ${ }^{2}$, remiten de diversas maneras a la experiencia con la tierra natal (Heimat) y al ocaso de ésta a través del desarraigo ${ }^{3}$. El hilo conductor de tales reflexiones es la indicación del peligro que representa el desarrollo aparentemente incontenible del pensar calculador y unilateral para la existencia humana y, en particular, para la pervivencia de la tierra natal. En el contexto de la preeminencia técnica global, la pérdida de la tierra natal no significa solamente el olvido de todo aquello que está implícito en los procesos de transmisión material de una tradición cultural particular, sino fundamentalmente la negación de la posibilidad misma del despliegue y protección de lo propio. La pérdida de lo propio tendrá como consecuencia directa, aunque a primera vista imperceptible, aún a la mirada de Heidegger, el camino hacia la homogenización que disuelve la diferencia ${ }^{4}$ y con ello, irremediablemente, la imposibilidad del primado de la ética en la existencia humana, si ésta verdaderamente puede estar fundada en el vínculo de "lo propio" con el Otro considerado como extraño (das Fremde) $)^{5}$. Tanto la pérdida del arraigo como el posible debilitamiento ético en el sentido antes señalado, tienen sus raíces en el predominio universal del pensar técnico-unilateral, fruto del olvido del ser por la carencia de un preguntar que indague por su sentido y por el carácter esencial de la diferencia.

El énfasis puesto sobre el fenómeno de la tierra natal no trata, sin embargo, de postular una defensa acérrima de la permanencia ciega en el pasado (Vergangenheit), sino más bien de resaltar aquello que constituye la esencia de nuestra propia experiencia: el cuidado (Pflege) de la diferencia, que corresponde al sentido más propio (eigentlich) de la existencia humana en tanto proyecto arrojado. Se trata, entonces, del cuidado de lo ontológicamente constitutivo y no solamente

\footnotetext{
2 Este término es usado por Heidegger en relación con la tierra natal a partir de 1969 en su "Dankansprache" en Messkirch (2000b, pp. 711-713). Cf. también "Ein Grusswort für das Symposion in Beirut" (2000b, pp. 742-743); "Grusswort anlässlich des Erscheinens von Nr. 500 der Zeitschrift Risô" (2000b, pp. 744-745); "Grusswort von Martin Heidegger" (1983a, p. 243). Es mencionado en otro contexto en Die Grundbegriffe der Metaphysik. Welt - Endlichkeit - Einsamkeit (1983b, p. 535). ${ }^{3}$ Cf. entre otros: Gelassenheit (1992); "700 Jahre Messkirch" (2000b, pp. 574-582) y "Dankansprache am 26. September 1969 in Messkirch" (2000b, pp. 711-713).

4 Esto quiere decir, usando términos de Heidegger, la preeminencia de la "identidad" vacía (Gleichheit) sobre la mismidad (Selbigkeit), rica en diferencias.

5 Pienso aquí en la concepción ética de Levinas y en los resultados de la dilucidación heideggeriana de los himnos de Hölderlin 'Andenken' (1980, pp. 79-151; 1982) y 'Der Ister' (1984). Si bien no se puede afirmar categóricamente la existencia de una ética en el abordaje heideggeriano de la experiencia con la alteridad, si es posible observar en las mencionadas dilucidaciones de la poesía de Hölderlin que el reconocimiento de lo propio pasa por el encuentro con lo extraño. En esta prevalencia del Otro coinciden, a pesar de todas sus diferencias fundamentales, la propuesta ética levinasiana y la reflexión heideggeriana mediada por el análisis de Hölderlin.
} 
de lo culturalmente valioso que, precisamente, adquiere su carácter en relación con lo constituyente de una experiencia primigenia con la diferencia. Este es el punto de encuentro, ontológicamente fundado, entre la experiencia de lo propio y la experiencia ética considerada a partir de la presencia de lo extraño. 'Lo propio', que no es excluyente en la filosofía de Heidegger6, y en tal sentido rebate desde dentro las interpretaciones políticas que tendenciosamente definen su pensamiento como una introducción nazi en la filosofía, se convierte en la contraposición ética a un mundo caracterizado por la tendencia cada vez más fuerte a la unificación "política", económica y "cultural".

La reflexión heideggeriana en torno al peligro que implica el desarraigo ${ }^{7}$ debe ser entendida fundamentalmente como una meditación acerca de la esencia de la civilización mundial y sus profundas raíces en la metafísica de occidente. En su saludo de agradecimiento a los organizadores del Simposio celebrado en Beirut en su honor, Heidegger expresa claramente este vínculo entre metafísica y civilización mundial: "La discusión con la metafísica, que de tal manera nos acosa, no es una anticuada polémica en contra de sus doctrinas acerca de Dios, el mundo y los hombres. Más bien, y ante todo, ella trae ante la mirada de un pensar meditativo los rasgos ontológicos fundamentales de la civilización mundial tecnológico-científica"8. Esta es la razón por la cual pensar en torno al problema de la tierra natal y su relación con la civilización mundial no significa de ninguna manera apartarse de la

\footnotetext{
${ }^{6}$ Remito al concepto de mismidad como expresión de una perspectiva no autoreferencial de lo que en términos generales podemos denominar 'lo propio'. Cf. por ejemplo, "Bauen Wohnen Denken" y "Das Ding” (2006a, pp. 151 ss. y 179 ss., respectivamente). También "Aletheia" (2006a, pp. 278-279).

7 Es importante diferenciar en la filosofía de Heidegger por lo menos dos tipos básicos de desarraigo, que permiten matizar su reflexión en torno a la nostalgia y al regreso a casa: a) uno de tipo marcadamente cultural, que se expresa en el abandono de lo propio del entorno o de la tierra natal en su acepción cotidiana, y que se puede apreciar, por ejemplo, en lo señalado en Gelassenheit (cf. 1959, p. 17), en la dilucidación de la poesía dialectal de Johann Peter Hebel y de los himnos de Hölderlin ya mencionados, pero también en su crítica al modo de vida citadino, tal como se puede observar en su texto apologético de la provincia y en las alocuciones festivas ya citadas; b) uno de carácter estructural, que se constituye en el telón de fondo del primer desarraigo y permite entender la imposibilidad de una apropiación definitiva de lo propio. Este desarraigo fundamental está señalado en Ser y tiempo: a) en relación con la tradición y la historicidad (Geschichtlichkeit), a través de la noción 'carencia de fundamento' (Bodenlosigkeit); b) en referencia a la habladuría (Gerede) por medio del concepto 'desenraizamiento' (Entwurzelung) y, c) directamente vinculado a la angustia (Angst) con los términos 'Unheimlichkeit' (desazón) y 'Un-Zuhause' (fuera de casa) (1977a, pp. 21, 170 y 188 s. respectivamente). Si bien el desarraigo estructural es la condición de posibilidad del 'estar fuera de casa' en sentido cultural, éste puede ser abordado, sin embargo, desde un marco de referencia propio, sin que se caiga con ello en un error de procedimiento. Es posible el retorno a casa desde lo extraño, sin que ello signifique en modo alguno una superación del desarraigo constitutivo de la experiencia humana. Éste desarraigo constitutivo es, precisamente, la causa de la búsqueda de lo propio a través de lo extraño, resaltado por Heidegger en su análisis de los himnos de Hölderlin. El arraigo en lo propio de la tierra natal no contradice, entonces, la esencia desarraigada de la existencia.

8 "Ein Grusswort für das Symposion in Beirut" (2000b, p. 742).
} 
reflexión filosófica para entrar en el ámbito de las apologías políticamente conservadoras del pasado, sino, de manera diferente, meditar acerca del presente desde las categorías filosóficas propias de la ontología fenomenológica heideggeriana. Se trata, en última instancia, de la pregunta por la esencia del habitar humano en medio del primado del pensar calculador, que se hace unilateral y progresivamente universal en la experiencia actual de un mundo globalizado.

\section{El sentido de la tierra natal}

La pregunta por el sentido de la tierra natal nos conduce más allá de la definición acostumbrada de ésta como lugar en el que se nace y se tiene residencia ${ }^{9}$. Heidegger mismo, quien a lo largo de su obra parece dejar entrever en más de una ocasión que comparte esta identificación de la tierra natal con un lugar geográfico específico ${ }^{10}$, supera esta limitada concepción al rebasar la delimitación espacial de ésta para vincularla estrechamente con la experiencia del desarraigo y, a través de ella, con el habitar del hombre en medio del predominio técnico que denota, por su parte, un estado particular del olvido del ser (Seinsvergessenheit).

El simplismo exacerbante con el cual el sentido común y la manipulación política determinan el significado de la tierra natal -que puede derivar fácilmente en una defensa apasionada y ciega de lo propio considerado en términos de patria (Vaterland) - es superado en la reflexión filosófica heideggeriana a través de la pregunta por el sentido de la experiencia del "encontrarse en casa"11. Esta experiencia no coincide, por supuesto, con el hecho de hallarse en un lugar que se considera y se siente como hogar ni con la identificación exclusiva de este lugar con la región o país de origen. El primer caso es controvertido, en primera instancia, por el hecho de que la tierra natal puede ser experimentada de manera especial en la nostalgia (Heimweh), es decir, precisamente en la ausencia y la lejanía del lugar de origen ${ }^{12}$ $\mathrm{y}$, en segundo lugar, por el carácter estructuralmente determinado del desarraigo humano ${ }^{13}$, que imposibilita la apropiación (Aneignung) de aquello que consideramos como propio (eigen). Este doble matiz de la experiencia del "encontrarse en

\footnotetext{
9 Cf. Deutsches Wörterbuch (Grimm, J. y Grimm, W., 1956, pp. 864 ss.).

10 Cf. entre otros, "Schöpferische Landschaft: Warum bleiben wir in der Provinz?" (1983a, pp. 9-13); "Dank an die Heimatstadt Messkirch", "700 Jahre Messkirch" y "Verlust der Heimat" (2000b, pp. 558, 576 y 634-637, respectivamente).

11 Esta pregunta está dirigida no solamente a la experiencia de lo natal en sentido particularista, sino también al sentido mismo del habitar en el contexto del mundo actual. Por esta razón excede la simple inquietud por el lugar de origen y su conservación.

12 Cf. por ejemplo, "Die Sprache Johann Peter Hebels" (1983a, pp. 123-124) y “700 Jahre Messkirch”, (2000b, p. 578).

13 Cf. nota No.7.
} 
casa" permite relativizar las lecturas conservadurizantes del arraigo así como las versiones de la tierra natal que tienden a identificarla con un ideal (cf. Marten, 1980, pp. 151 ss.) y que, de una $u$ otra manera, inevitablemente conducen a una interpretación política totalitaria del pensamiento de Heidegger.

Esta es la vía seguida por Rainer Marten, por ejemplo, en su interpretación del concepto de tierra natal en la filosofía de Heidegger. Partiendo de su caracterización de la lectura heideggeriana del desarraigo estructural del hombre y de la carencia de tierra natal como una patología fundada en la nostalgia por la "madre perdida" (cf. pp. 136 ss.), Marten concebirá la filosofía de Heidegger, junto a la del principio esperanza de Ernst Bloch, como una ideología filosófica (p. 139). Las razones para hacer esta aseveración son expuestas a través de una estructura lógicamente válida en sus conexiones causales, aunque bien vale la pena preguntar si también son totalmente válidas las consecuencias extraídas de estas razones y si realmente, por lo menos en el caso de Heidegger, el destinatario de las críticas es el adecuado: ante la situación de desarraigo en que se halla el hombre contemporáneo, y presionada por la profunda nostalgia que la carencia de tierra natal produce, se hace acuciante la necesidad de superar la pérdida de lo propio. Se desencadena, entonces, el deseo por recuperar lo previamente perdido, que tiene como consecuencia la negación del presente a favor de un futuro ideal en el que es posible superar dicha carencia y en el que, paradójicamente, se pretende recuperar el pasado como auténtica expresión de lo propio. En este movimiento de superación de la pérdida, el ideal queda al servicio de lo ya sido (Vergangenheit) y, con ello, de su conservación. Esta es la consecuencia fundamental: lo que en principio parece ser una superación no es más que un simple movimiento de retorno en el tiempo y, de esta manera, al pasado. El futuro puede convertirse, entonces, en la expresión de un salto atrás, a lo ya sido, que es apropiado como un ideal en el que se pretende conservar un origen petrificado.

Esta interpretación se alimenta también de la identificación de la tierra natal con un lugar geográficamente definido y, adicionalmente, con una pretensión de universalizar lo propio como paradigma para metas ajenas. Independientemente del hecho de que el arraigo sea visto a partir de la contraposición con el desarraigo implícito en la vida citadina -como se aprecia por ejemplo en el corto escrito de 1933 "Schöpferische Landschaft: Warum bleiben wir in der Provinz" (cf. 1983a, pp. 913) - o como contraparte del desenraizamiento producto del predominio universal del cálculo técnico -señalado expresamente por Heidegger 22 años después en "Gelassenheit"- esta lectura del significado de la tierra natal enfatiza el carácter espacial de ésta, como una forma de resaltar sus limitaciones y, desde esta versión, su carácter escatológico. En cualquiera de los dos casos -que en términos de Wolfgang Welsch pueden ser entendidos como dos puntos de partida que Heidegger emprende en su reflexión (cf. 1985, pp. 95 ss.): el primero catalogado como una posición que promueve la conservación y defensa de una tierra natal intacta $\mathrm{y}$, el 
segundo, como una concepción histórico-ontológica y progresista de la misma, que parte de concebir el desarraigo como experiencia universal (cf. p. 96)- Marten pone el acento sobre la propia experiencia "cultural" de Heidegger (cf. 1980, p. 139) para señalar el lugar y el "nombre" desde el cual se pretende fundar y sustentar la escatología del arraigo (cf. p. 143 y 150).

En la determinación de un nombre particular para la experiencia del arraigo se integran, entonces, los dos rasgos característicos de la concepción cotidiana de la tierra natal, que son básicamente los mismos que sustentan el concepto cerrado y políticamente usufructuado de "patria". El nombre particular que se otorga a la experiencia de lo propio (das Eigene) permite la unificación del "nosotros" en una especie de mónada del "común acuerdo" latente e indiscutible, cuyo contenido es presupuesto en la pervertida esencia formal del término "patria". El nacionalismo exacerbado, la discriminación cultural y el totalitarismo implícito en ellos, no son más que el desarrollo al extremo de dicha identificación del significado de 'tierra natal' con un nombre y un lugar. En este fenómeno encuentra toda su justificación la crítica de Marten a los conceptos ideologizados de 'carencia de tierra natal' (Heimatlosigkeit) y 'enraizamiento' (Verwurzelung), que se oponen a su propia concepción, en la que lo natal es definido en términos de una dimensión de la vida práctica que se comparte de manera satisfactoria ${ }^{14}$.

No obstante la validez de esta crítica, la identificación de la tierra natal con un nombre propio no es en la filosofía de Heidegger tan evidente como parece ser a los ojos de Marten. Si bien es cierto que en algunos de los artículos, conferencias y alocuciones de su época tardía, Heidegger enfatiza el carácter situado de la experiencia del arraigo, también lo es el hecho de que en estos mismos textos se encuentran claros indicios que permiten relativizar la deificación del propio lugar de origen y con ello su posible carácter absoluto, excluyente y universalizable. Es así como en un plexo de sentido se cruzan en las reflexiones de Heidegger "versiones" a primera vista contrarias de la tierra natal, en el que confluye el apego a las raíces y al origen en su sentido más común y extendido, con la puesta en duda de la esencia geográficamente situada de la experiencia del arraigo, que señala una actitud de reconocimiento de otras "tierras natales" y resalta la esencia estructural del desarraigo. De esta manera la tierra natal es provista de un carácter global, que va más allá de toda definición reducida del arraigo a una determinación local referida a un pueblo, a una región, a un país o a un conjunto de costumbres y experiencias compartidas en el lugar de origen.

Uno de los más interesantes ejemplos de esta "des-espacialización" de la experiencia de la tierra natal está presente en la alocución de 1955 "Gelassenheit". Después de dejar la impresión de concebir la tierra natal como un simple lugar de origen geográfico (cf. 1959, pp. 11 s.), que se fortalece con una lectura apresurada

14 Cf. ibíd., pp. 141 ss. y 159. 
del verso varias veces citado de Johann Peter Hebel ${ }^{15}$, Heidegger va más allá de los límites impuestos por éste y se adentra en una concepción de la carencia de tierra natal muy ligada a la reflexión desarrollada en su dilucidación de la esencia de la técnica al concebir el desarraigo como un fenómeno epocal: "Muchos alemanes han perdido su tierra natal, debieron abandonar sus pueblos y ciudades, fueron expulsados del suelo natal. Muchos otros, que conservaron su tierra natal, emigraron, sin embargo, y cayeron en el ajetreo de las grandes ciudades, debieron asentarse en el desierto de las zonas industriales. Se volvieron extraños de su antigua tierra natal. ¿Y qué pasa con aquellos que permanecieron en ella? En muchos aspectos están aún más desarraigados que quienes han sido expulsados de su tierra natal [...] La pérdida del arraigo proviene del espíritu de la época [...]" (1959, p. 17. Las cursivas son mías).

Esta concepción, en la que se integran dos elementos característicos del primer punto de partida indicado por Welsch -el énfasis en el pueblo natal y la confrontación con la gran ciudad - con el marco general del segundo punto de partida, permite pensar la experiencia de la tierra natal en una acepción que no coincide con la versión tradicional, ligada a la ingenuidad de la vida cotidiana y al uso estratégico de 'lo propio' con fines políticos. La carencia de tierra natal, y con ello el concepto mismo de 'lo natal', está fundada en el espíritu de la época técnica y sus exigencias (cf. Heidegger, 1959, pp. 18 ss.) y no en el abandono espacial del lugar de origen. Esta época de predominio técnico, por su parte, al ser identificada con el modo de pensar que calcula y su pretensión de dominio universal sobre todo ente implica, en consecuencia, la postulación restrictiva de un sólo modo de "hacer y dejar venir" a presencia 16 .

Desde una perspectiva óntica, la carencia de tierra natal tiene su raíz en el desarrollo de una época caracterizada por el cálculo y la ausencia, cada vez más evidente, de la pregunta por el sentido de lo que es (besinnliches Denken). De esta manera la experiencia del desarraigo remite a un modo particular de la existencia humana en la que el hombre se entrega irreflexivamente al dominio del mundo bajo los cánones de la relación universal de medios y fines. Pero la invitación a permanecer en casa -realizada en algunas ocasiones con marcado acento provinciano y regionalista 17 - no debería ser considerada como el punto de partida de la reflexión heideggeriana en torno a la tierra natal, sino como una consecuencia lógica del proyecto fundamental que guió, en diversas formas y matices, toda la obra de Heidegger: la pregunta por el sentido del ser.

\footnotetext{
15 Cf. Gelassenheit (1959, pp. 16 y 28); "Hebel - Der Hausfreund" (1983a, p. 150); "Johann Peter Hebel" (2000b, p. 530).

16 Cf. "Die Frage nach der Technik" (2006a, pp. 5-36).

17 Cf. nota No. 10.
} 
Pasar por alto esto tiene como consecuencia inmediata no solamente la limitación de la problemática aquí tratada al ámbito de una reflexión cultural, política o ideológica -centrada fundamentalmente en el concepto de arraigo concebido en su acepción geográfica, política o cultural-, sino también en alguna medida la fragmentación de la obra de Heidegger en temáticas aisladas, sin un hilo conductor que permita una interpretación coherente de las múltiples problemáticas planteadas en ella. Es en esta dirección que la pregunta por la tierra natal no debe ser entendida como un punto de partida en sí mismo, sino como un punto más de llegada de la pregunta fundamental por el sentido de lo que es; en esto consiste, precisamente, la esencia del indagar meditativo que caracteriza al pensar del mismo nombre, que Heidegger opone al pensar que calcula. La pregunta por la tierra natal es, entonces, una pregunta de carácter ontológico-estructural, que implica directamente la constitución de la existencia humana y no solamente el modo de vida de un pueblo o región en particular ${ }^{18}$; es la pregunta esperada ante el desarraigo constitutivo de la existencia humana, ligado estructuralmente al olvido del ser (Seinsvergessenheit) ${ }^{19}$. En "Brief über den Humanismus" podemos observar la relación que Heidegger establece entre carencia de tierra natal y olvido del ser y, con ello, el fondo ontológico fundamental en el que se sustenta toda pregunta por el sentido de lo natal. No se trata, entonces, en su origen, de un indagar por un lugar específico que pretende ser defendido de las influencias externas, sino de la pregunta esencial por nuestra cercanía, en tanto apertura, al ser: "Esta palabra [Heimat] es pensada aquí en un sentido esencial, no patriótico ni nacionalista, sino en el sentido de la historia del ser. La esencia de la tierra natal ha sido nombrada al mismo tiempo, sin embargo, con el propósito de pensar el desarraigo (Heimatlosigkeit) del hombre moderno a partir de la esencia de la historia del ser" (1976, p. 338).

\section{Tierra natal y despliegue de lo propio}

La complejidad del concepto de tierra natal en la filosofía de Martin Heidegger no se deja explicar a través de la simple oposición entre una concepción particularista-regional del apego al lugar de origen y una universalista-global fundada en la toma de distancia ante el fenómeno del predominio técnico en el mundo contemporáneo. No se puede entender tampoco como una mera evolución de su pensamiento, que lo llevaría de la primera posición, defendida en sus escritos iniciales, a la segunda, desarrollada en su filosofía posterior. Esta linealidad en la interpretación no lograría explicar la permanente y simultánea presencia de las dos posiciones en momentos diversos de la obra de Heidegger ni tampoco el carácter integral de su

18 Cf. nota No. 7.

19 Cf. "Brief über den Humanismus" (1976, p. 339). 
tratamiento. Wolfgang Welsch señala en un primer momento de su reflexión la oposición entre particularismo y universalismo, basado inicialmente en dos textos "representativos" del abordaje heideggeriano al problema. Por una parte se encuentra la perspectiva histórico-regionalista expresada en la corta reflexión de 1933 "Warum bleiben wir in der Provinz"; por otra parte, la perspectiva ontológico-universalista desarrollada en su escrito de 1946 "Brief über den Humanismus" (cf. Welsch, 1985).

No se trata aquí, sin embargo, como ya ha sido mencionado, de la superación de una perspectiva por otra. Esto puede ser corroborado fácilmente por la presencia de cada una de estas perspectivas en escritos posteriores de la obra heideggeriana, en los que debería presupuestarse -si de una simple evolución se tratara- la ausencia de la concepción regionalista, propia de su etapa inicial. Welsch llamará la atención sobre el retorno de la posición particularista en el corto escrito de 1949 "Der Feldweg”20, y de la concepción universalista en Gelassenheit (cf. 1985, pp. 95 ss.). Pero más allá de una simple contraposición de dos ópticas radicalmente opuestas, se trata de un posible vínculo de las mismas en una visión compleja, que aparece explícitamente con mayor fuerza en alocuciones y artículos cortos posteriores al discurso rememorativo de Konradin Kreutzer. En este sentido coincido, entonces, enteramente con lo afirmado por Welsch, quien postula una perspectiva general, que surge de una relación de equilibrio entre las lecturas particularista-conservadora y la ontológico-universalista (cf. Welsch, 1985, pp.100 ss.).

Esta interpretación puede ser radicalizada, sin embargo, en dos aspectos. En primer lugar si se entiende la relación de las dos perspectivas como un obvio entrecruzamiento de los ámbitos óntico y ontológico de la experiencia de 'lo propio' que se expresa en la pregunta por la tierra natal y, en segunda instancia, si se puede mostrar que tal entrecruzamiento está ya por lo menos indicado desde el inicio mismo de la reflexión heideggeriana al respecto. Con esto quiero resaltar que no obstante el hecho de que la relación indicada por Welsch se expresa con mayor claridad en los años posteriores a la alocución festiva de 1955 -como por ejemplo en las reflexiones referidas por él (ibíd.): "Dank an die Heimatstadt Messkirch (27. September 1959) y "700 Jahre Messkirch (Ansprache zum Heimatabend am 22. Juli 1961)-, este entrecruzamiento ya está presente, sin embargo, en las primeras reflexiones de Heidegger. Es evidente que todo el entorno textual de "Schöpferische Landschaft: Warum bleiben wir in der Provinz" hace referencia al ámbito de vida campesina propio de la selva negra; la descripción de este entorno no puede ser más elocuente. No obstante, esta evidencia no puede ser tomada simplemente como una prueba irrefutable del centrismo regionalista de Heidegger y de su concepción conservadora y excluyente de la tierra natal, como si se tratara de una defensa acérrima del pro-

20 Cf. "Der Feldweg" (1983a, pp. 87-90). Habría que referir también a "Verlust der Heimat" y "Festansprache beim Heimatfest in Todtnauberg" (2000b, pp. 634-637 y 641-649). 
pio lugar de origen, concebido como un ideal que puede llegar a ser universalizable. Por el contrario, este corto texto integra de manera germinal los mismos elementos posteriormente desarrollados en escritos tardíos, en los que la literatura especializada reconoce una perspectiva no particularista de la pregunta por la tierra natal.

En primer lugar hallamos el carácter personal de la referencia a lo natal, que coincide con lo expresado en apartes de la alocución de 1955, de la que se puede citar, entre otros ejemplos: "Es suficiente si nos detenemos en lo que está cercano y meditamos en torno a lo más próximo: en torno a lo que nos concierne a cada uno de nosotros aquí y ahora; aquí: en este rincón de la tierra natal; ahora: en el acontecer mundial presente" (Heidegger, 1959, p. 16). Esta afirmación, que invita a pensar simultáneamente desde el arraigo a las propias raíces y desde el contexto general de la época contemporánea, y que señala a su vez un movimiento constante de oscilación entre la experiencia fáctica del estar en casa y la pregunta por la esencia del mundo regido por el pensar técnico, no es más que una clara indicación de la necesidad de abordar el fenómeno de la tierra natal desde una perspectiva compleja en la que confluyan en su indisoluble vínculo interno la experiencia de lo propio y la indagación filosófica por el sentido de todo lo que es ${ }^{21}$.

En segunda instancia podemos observar también el preludio del distanciamiento posterior de Heidegger ante el modo de vida citadino, que sirve de base a su convicción acerca de la imposibilidad de una auténtica soledad (Einsamkeit) reflexiva en el mundo de la celebridad (Berühmtheit) propiciado por la ciudad así como a la reiterada, $\mathrm{y}$ un tanto peyorativa, mención de la visión que puede tener el hombre de la urbe respecto al modo de vida campesina. Este distanciamiento inicial será el germen de lo que posteriormente significará para Heidegger la gran ciudad dominada por el incontenible apremio y productividad técnica 22 .

Hallamos un plexo similar, en el que coinciden la defensa de lo propio con la crítica a la gran ciudad y la descripción del auge técnico, en la ya referida "Ansprache zum 700 Jahre Messkirch" de 1961. Heidegger se pregunta aquí por la mejor manera de enfrentar lo inhogareño de la situación actual. Responde apoyando la conservación de la naturaleza y la transmisión (Überlieferung) de la tradición, pues estima que solamente es posible hacer frente a esta situación desde aquellas regiones campesinas y pequeñas ciudades que sean capaces de establecer límites ante la vida citadina en las grandes ciudades industrializadas (cf. 2000b, pp. 576577). ¿Es esto un retroceso que marca el retorno a la perspectiva regional-particu-

21 En esta misma dirección confróntese también, por ejemplo, "700 Jahre Messkirch", "Dankansprache", "Ein Grusswort für das Symposion in Beirut" y "Grusswort anlässlich des Erscheinens von Nr. 500 der Zeitschrift Risô", ya citados.

22 Cf. por ejemplo, "Bauen Wohnen Denken" (2006a, pp. 147 ss.); "Rede auf Hebel" (2000b, pp. 537 s.); "700 Jahre Messkirch" (2000b, pp. 575 s.); "Das Ding" (2006a, pp. 167 ss.) y Unterwegs zur Sprache (1985, pp. 198 s.). 
larista después de haber dado el paso a la acepción global del fenómeno del desarraigo y de la tierra natal? Podría responderse afirmativamente si se sostuviera aún la lectura que plantea dos momentos diversos e inconexos en el tratamiento de la experiencia de la tierra natal. Se trata, sin embargo, de dos énfasis complementarios del mismo fenómeno, que no podrían ser entendidos cabalmente uno sin el otro: la civilización mundial, como expresión del desarraigo producto del predominio técnico-científico, y la carencia de lo natal no son más que la negación de las posibilidades mismas de lo propio. Esto quiere decir que el mundo actual, que se entrega hasta en sus más mínimos detalles al pensar que calcula y su unilateralidad, deja de ser el lugar propicio para el despliegue del pensar que pregunta por el sentido de lo que es y, de esta manera, para el habitar en lo propio, que no puede ser reducido simple y llanamente al hecho de 'estar en un lugar'.

Con el predominio universal del pensar propicio al despliegue técnico en su vertiente moderna (Gestell) se promueve el olvido de aquel modo de pensar que está en capacidad de preguntar por el sentido de lo que es propio, es decir, por el sentido de la tierra natal. El ocaso de 'lo propio', por su parte, implicaría la imposibilidad del asombro ante lo extraño, que en la época en que predomina el cálculo se convierte en nada más que en lo inquietante (das Unheimliche). Pero de manera paradójica, solamente desde la escucha de lo inquietante del mundo técnico, que amenaza con su unilateralidad y universalismo, será posible reconocer lo propio que constituye la experiencia del hombre; en esto consiste tanto el carácter otorgado al Ereignis (cf. 2006, pp. 45 ss.) como el vínculo resaltado por Heidegger entre peligro y salvación ${ }^{23}$. De esta manera, en la pregunta por 'lo propio' y en la defensa de éste, se hace manifiesta la preocupación por el peligro que representa el olvido de la pregunta por el sentido y la amenaza que significa el dominio total del pensar técnico-calculador. Es en este contexto que la perspectiva regional-particularista en torno a la tierra natal y la concepción global de la misma no deben ser vistas como propuestas independientes o diametralmente opuestas, sino como caras de un único modo de proceder en el que concurren simultáneamente la apología de lo propio y el llamado de atención ante lo inquietante e inhogareño (das Unheimische) del mundo actual. La confianza depositada en la provincia ante las seductoras atracciones de la gran ciudad y la exhortación al auténtico habitar humano frente al simple hecho de encontrarse en un lugar de residencia en el que lentamente se convierte el mundo bajo los designios del pensar técnico ${ }^{24}$, son la expresión conjunta del esfuerzo por mantener abierta la pregunta por el sentido de todo lo que es. En este punto coinciden las dos perspectivas de la tierra natal y del desarraigo, concebidas inicialmente como incompatibles.

23 Cf. por ejemplo "Die Frage nach der Technik" (2006a, pp. 29 ss.); "Heimkunft / An die Verwandten" (1980, p. 21); “Wozu Dichter?” (1977b, pp. 296 ss.).

24 Ver "Hebel - Der Hausfreund" (1983a, pp. 146 ss.). 
Esta simultánea referencia a lo que aparentan ser dos puntos de partida diferentes, con una tendencia a ser interpretados desde una lectura lineal y evolutiva -la perspectiva regional superada por una concepción global, fundada en una actitud crítica ante el desarrollo prepotente de la técnica y la ciencia- está claramente señalada también en la alocución conmemorativa de los 700 años de Messkirch: la pregunta por el sentido de la tierra natal en su acepción regionalista y la pregunta por el sentido (esencia) de la técnica en el contexto de su relación con el peligro que significa la caída del hombre en la absoluta carencia de tierra natal, son planteadas aquí como una unidad con dos caras complementarias. Se sostiene de esta manera la tesis de la mutua dependencia en que se hallan la indagación por lo propio y la pregunta por lo inhóspito (Umheimliche) de la preeminencia del cálculo y la unilateralidad. Ante la pregunta por el futuro de su pueblo natal, Heidegger sostiene: "[Messkirch] Estará enredada en la red de la época técnica. La pregunta que se plantea no solamente a esta ciudad ni solamente a nuestro país, ni exclusivamente a Europa, sino a los hombres de esta tierra, es si bajo el dominio de la técnica moderna y con las transformaciones del mundo producidas por ella, es posible aún en cualquier sentido la tierra natal" (2000b, p. 578).

Como puede observarse en este caso, la pregunta de Heidegger por la tierra natal no pretende universalizar la experiencia particular de lo propio. Es evidente que esta pregunta implica al hombre en general y su necesidad de arraigo en medio de la unilateralidad homogeneizante del pensar que calcula 25 . Esta no es, sin embargo, una característica exclusiva de la filosofía heideggeriana posterior a los años 50 , sino una tendencia general de su reflexión en torno a la tierra natal. Tanto la referencia particular que se hace al arraigo en la reflexión de 1933 como el distanciamiento al modo de vida de la gran ciudad, ya mencionados, así lo atestiguan. El uso ya temprano del pronombre posesivo y de la experiencia personal26, del artículo indefinido para referirse a la experiencia del propio mundo 27 , no deben ser interpretados como una expresión del encapsulamiento dentro de las propias fronteras, sino como la obvia manifestación del carácter arrojado de la existencia humana. Con ello no se excluye de alguna manera la experiencia de 'lo propio' de otros "mundos" ni se universaliza la propia 28 , sino que a través de ésta se resalta la importancia del arraigo frente al vacío producido por la universalización de un único modo de pensar.

\footnotetext{
25 En este sentido la pregunta ¿es aún posible la tierra natal? está referida fundamentalmente a la experiencia del habitar humano en medio del contexto actual del predominio técnico. Cf. "700 Jahre Messkirch" (2000b, p. 578) y no exclusivamente al interés por la conservación cultural de un lugar particular.

26 Cf. "Schöpferische Landschaft: Warum bleiben wir in der Provinz?" (1983a, pp. 9 ss.).

27 Cf. por ejemplo, "Hölderlin und das Wesen der Dichtung" y "Wie wenn am Feiertage" (1980, pp. 40,46 y 64 respectivamente).

${ }^{28}$ La tierra natal no es identificable con un pueblo, una región o una nación específica -con ello se caería de manera evidente en una acepción totalitaria y fundamentalista de la propia experiencia- ni
} 
Para el Heidegger de "¿Por qué permanecemos en la provincia?” es la ciudad el terreno propicio para el rápido desarrollo de la tendencia hacia el vacío que nos deja el despliegue avasallador del cálculo. Esta concepción implícita es la que posteriormente se hace evidente en la crítica expresa a los medios de comunicación ${ }^{29}$, al modo de "habitar" del hombre contemporáneo en medio del aparente acortamiento de las distancias y el logro cada vez mayor de bienestar ${ }^{30}$, al uso de las máquinas de hablar y calcular ${ }^{31}$, a la postulación oculta de un pretendido lenguaje universal y a la negación de la riqueza del propio lenguaje ${ }^{32}$, etc. Calificar esta actitud como un retroceso romántico, acampesinado y conservador no solamente pasa por alto la pregunta por el significado de aquello que entendemos acríticamente como 'avance' y progreso, sino también el contexto general de la filosofía heideggeriana y su concepción de la existencia humana como proyecto (apertura a la posibilidad) arrojado. En el marco de una reflexión en torno al sentido de la tierra natal, esta concepción del Dasein como proyecto arrojado podrá ser leída en términos de "posibilidad arraigada". Esta reinterpretación permitirá, por su parte, encontrar importantes consecuencias de la pregunta por lo natal para el ámbito de una ética ontológicamente fundada.

\section{La condición ontológica de la civilización mundial}

Como ya lo he afirmado, Heidegger llama la atención acerca del fenómeno cada vez más extendido de la falta de arraigo a lo propio. Lo hace a través de diversos acentos que le permiten mantener el hilo conductor de su lectura ontológica de la

\footnotetext{
tampoco con pequeñas unidades geográficas o culturales como un poblado, un lugar de la ciudad o una forma de vida particular (cf. Welsch, 1985, pp. 92 s.). Heidegger no pretende, entonces, universalizar su experiencia de la tierra natal, sino de manera diferente llevar a cabo una defensa de lo propio frente a pretensiones externas de universalización y homogenización de la existencia humana. Con ello termina haciendo una defensa filosóficamente sustentada de la posibilidad de la diferencia implícita en el fomento de lo natal. Todo esto tiene como marco de referencia la pregunta por el ser y, de esta manera, la apertura del hombre a la posibilidad. La pregunta por la tierra natal es, finalmente, la pregunta ontológica por la esencia y el origen. Heidegger, acudiendo a Hölderlin y su búsqueda de la esencia de lo propio, afirma en "Brief über den Humanismus": "De manera alguna él busca esto en el egoísmo de su pueblo. Lo ve, más bien, a partir de la pertenencia al destino de Occidente (Geschick des Abendlandes). Solo que Occidente tampoco está pensado en sentido regional como lo opuesto (Occident) a oriente, no es pensado simplemente como Europa sino, desde la perspectiva de la historia universal, desde la cercanía al origen" (1976, pp. 338 s.).

29 Cf. entre otros, Gelassenheit (1959, p. 17); "Die Sprache Johann Peter Hebel” (1983a, p. 136) y "Rede auf Hebels" (2000b, p. 535).

30 Cf. "Bauen Wohnen Denken" y "Das Ding" (2006a).

31 Cf. "Der Satz vom Grund" (1997, p. 145); "Hebel - Der Hausfreund" (1983a, pp. 148 s.) y "Johann Peter Hebel" (2000b, pp. 531-532).

32 Cf. los textos ya referidos en torno a la poesía dialectal de Johann Peter Hebel.
} 
existencia humana. ¿Cuál podría ser entonces la razón para abandonar su pregunta fundamental por el sentido de lo que es, precisa y tal vez exclusivamente, cuando se trata de preguntar por el sentido de la tierra natal, para de esta manera caer en un simplismo particularista y meramente apologista de su propio entorno histórico-cultural? Considero que aún en sus escritos y alocuciones más aparentemente apologéticos y provincianos, la pregunta por el sentido estructural del fenómeno de lo propio es el hilo conductor de su reflexión. En este sentido, no se trata, entonces, de una indagación restringida a la ingenua pregunta por las razones de la pérdida de lo propio ( $\boldsymbol{s} \boldsymbol{u}$ tierra natal) y a la defensa del retorno o permanencia anquilosada en ésta, sino de una meditación filosófica en torno al carácter estructural de tal pérdida y al significado de ésta para la existencia humana. Esta es la razón fundamental de la pregunta por el papel de la técnica y su modo específico de pensar en el proceso de desarraigo que caracteriza al hombre contemporáneo.

Con la relación que se establece entre la técnica y el fenómeno del desarraigo se hace evidente que la experiencia de la tierra natal, así como su carencia, remite ineludiblemente a la muy conocida diferenciación planteada por Heidegger entre el pensar que medita y el pensar que calcula. Lo fundamental de esta diferenciación estructural de los modos de pensar se encuentra, a mi modo de ver, en la relación que cada uno de ellos puede tener con la determinación de la existencia humana como apertura a la posibilidad. Mientras que el pensar meditativo, en tanto pensar que pregunta por el sentido, se vincula intrínsecamente con la actitud de apertura al misterio, el pensar técnico-calculador se caracteriza por su unilateralidad y sus pretensiones de universalidad.

La pérdida del arraigo es la expresión del despliegue avasallador del pensamiento técnico y, con ello, de la unilateralidad que se hace universal en dicho despliegue. La carencia de tierra natal no es entonces un asunto referido exclusivamente a la pérdida de una cultura y una historia particular, sino un fenómeno que compromete la constitución misma de la existencia humana. Es por esta razón que la pregunta por el sentido de la tierra natal, e incluso la defensa acérrima de 'lo propio', no puede ser apresuradamente menospreciada como una ingenua reflexión provinciana, pasando por alto que su origen está profundamente enraizado en la pregunta filosófica por la estructura ontológica del presente y, de esta manera, por el ser de la experiencia humana en la era científico-técnica. La pregunta por la tierra natal conduce la meditación heideggeriana hacia el fenómeno del desarraigo y éste, por su parte, nos lleva hacia la esencia de la técnica, convertida en fenómeno global. Precisamente desde el análisis del fenómeno de la técnica moderna surge con mayor vigor la pregunta por el sentido de 'lo propio' como posibilidad de resguardo ante el predominio de lo 'mismo' (Gleichheit).

Este es precisamente el peligro generado por el pensar que calcula: que aquello que denominamos tierra natal se desvanezca y desaparezca en 'lo mismo' e 'idénti- 
co'33. Alertar ante este peligro no significa propender solamente por la conservación de particularidades culturales y tradiciones olvidadas, sino pretender salvar (dejar ser) lo propio de la existencia humana en tanto apertura a la posibilidad. El mayor peligro que representa el predominio técnico es la caída de la experiencia humana en la uniformidad (Gleichförmigkeit) y la indiferencia (Gleichgültigkeit), pues lo que finalmente está en juego con ello -aún sin que Heidegger lo señale de manera explícita- es la posibilidad misma de la conservación de la diferencia ${ }^{34}$.

Lo afirmado en "Gelassenheit" acerca del vínculo que se puede establecer entre el predominio técnico, la carencia de pensar (Gedankenlosigkeit) y el desarraigo (Bodenlosigkeit) se expresa en "Die Frage nach der Technik" y en escritos posteriores, a través de las figuras del Gestell y la Weltzivilisation: el Gestell es concebido como la fase final de la civilización mundial. Con ello no se hace referencia, sin embargo, a un producto de la voluntad humana, sino a un acontecimiento propio del despliegue histórico del ser. Hemos llegado a esto en medio de un proceso histórico que nos excede y se nos impone sin miramientos ${ }^{35}$. Pero la civilización mundial es simultáneamente desarraigo y pérdida de lo propio. Se completa de esta manera un entramado que termina asfixiando la existencia humana: "La carencia de tierra natal es un destino del mundo (Weltschicksal) en la estructura de la civilización mundial. Parece como si esta civilización mundial trajera consigo un ensombrecimiento de la existencia, que el mismo hombre moderno no ha producido, (sino) en el que él más bien ha sido 'destinado' (geschickt)" (2000b, p. 713).

Este entramado constituye, finalmente, el peligro (Gefahr) advertido por Heidegger de diversas maneras: la disponibilidad de todo lo ente (2000b, p. 743), la desaparición de la tierra natal (2000b, pp. 575-576) y del pensar que medita ${ }^{36}$, la incapacidad para afrontar las exigencias del pensar que calcula, etc. El rasgo predominante de este fenómeno es, sin lugar a dudas, su tendencia hacia la "uniformidad" (Gleichförmigkeit) ${ }^{37}$. Podemos constatar esta inclinación en el despliegue homogenizador del Gestell y su propensión a universalizar la exigencia que se hace a todo ente como reserva (Bestand). Esto coincide con la concepción del pensar que calcula como un modo de pensar unilateral (einseitiges Denken), cerrado al misterio y a la pregunta por el sentido de lo que es. Enredado en medio de este entramado

33 Cf. "700 Jahre Messkirch" (2000b, pp. 575 s.).

34 "Ein Grusswort für das Symposion in Beirut" (2000b, p. 743): "Probablemente la civilización mundial moderna lleva a cabo el tránsito a la fase final del destino epocal del ser, en el sentido de la determinación de éste como disponibilidad incondicional de todo ente, que incluye al ser humano. Por esta razón es necesario por ahora indagar por el origen de este peligro y, en consecuencia, avistar su alcance. Esto exige, sin embargo, plantear la pregunta por lo propio del ser como tal".

35 Cf. "Die Frage nach der Technik" (2006a, pp. 5-36) y "Der Satz der Identität" (2006, pp. 42 ss.).

36 Cf. "Ein Grusswort für das Symposion in Beirut" (2000b, p. 742) y "Grusswort anlässlich des Erscheinens von Nr. 500 der Zeitschrift Risô" (2000b, p. 744).

37 Cf. de manera paradigmática: "Überwindung der Metaphysik" (2006a, p. 95). 
experimentamos el ocaso de la tierra natal y del arraigo: lo que se oculta aquí en la relación de este ocaso con la civilización mundial y el predominio del cálculo es un profundo nexo de carácter estructural entre el avance arrasador de la homogenización técnica -que en sentido estricto significa universalización política, económica y cultural ${ }^{38}$ - y el repliegue de 'lo propio'. Se trata en última instancia de un proceso en el que, con la disolución imperceptible de 'lo propio', la homogenización mundial pone en peligro también una auténtica apertura a lo extraño, que por antonomasia es trascendente a las pretensiones de apropiación (Beherrschung) y explicación totalizantes.

Esta consecuencia ética del predominio técnico del mundo y del correspondiente despliegue del cálculo y la exigencia de disponibilidad va mucho más allá de lo explícitamente sostenido por Heidegger, pero no excede en ningún momento lo implícita y coherentemente abierto por su reflexión. La pregunta por la tierra natal es en esencia, entonces, una pregunta por el sentido mismo de 'lo propio' (das Eigene) y, de esta manera, una referencia obligada a la experiencia 'del Otro' en tanto extraño (das Fremde) ${ }^{39}$. La indisoluble mutua pertenencia de 'lo propio' y 'lo extraño' permite entender el carácter primario de la "presencia" del Otro en la experiencia de lo propio: estructural, ontológicamente pensado, el extraño es la condición de posibilidad de la experiencia de lo propio, pues no es posible una experiencia de "sí mismo" ajena a la interpelación del Otro, que me constituye desde su Otredad. El camino hacia la apropiación de lo propio (Aneignung des Eigenen) ${ }^{40}$, que pasa por el encuentro con el extraño, de acuerdo con lo sostenido por Heidegger en su dilucidación de la poesía de Hölderlin, nos enseña algo fundamental: 'lo propio' se constituye como tal sólo 'en' y 'a partir' de la diferencia con 'lo Otro'. Desde esta perspectiva, 'el Otro' es 'lo propio' que se reconoce en nuestra presencia de extraños que lo interpelan. No se trata, sin embargo, de un intercambio de roles conmutables o transferibles, sino de la experiencia misma de la mutua y simultánea pertenencia de 'lo propio' y 'lo extraño'. Soy simultáneamente un 'propio-extraño' en relación permanente y dependiente con un 'extraño-propio' que me interpela y constituye. 'Lo propio' sin 'lo Otro' sólo es posible de ser imaginado en términos de un absoluto vacío y cerrado en sí mismo; una especie de mecanismo autosuficiente de procesos y engranajes absolutamente independiente y formal.

Esto es lo que está en el fondo de la aparentemente ingenua pregunta de Heidegger por el arraigo: ¿qué espera al hombre después de la muerte de lo propio

38 Cf. por ejemplo, "Dankansprache" (2000b, pp. 711 ss.).

39 Cf. al respecto el significado del "extraño" en la dilucidación heideggeriana de Hölderlin en “Andenken" (1980, pp. 79-151); Hölderlins Hymne »Andenken« (1982) y Hölderlins Hymne »Der Ister « (1984). Confróntese también la concepción ética levinasiana y el valor dado en ésta al "Otro" en tanto trascendente (Levinas, 2008).

40 Cf. “Andenken” (1980); Hölderlins Hymne »Andenken« (1982) у Hölderlins Hymne »Der Ister« (1984). 
inherente a la carencia de tierra natal? Con la muerte de lo propio no desaparecen simplemente las tradiciones y costumbres que nos diferencian en el plano más elemental ${ }^{41}$, sino la posibilidad misma de lo extraño, es decir, de 'lo Otro', y con ello de la diferencia. Sin 'lo propio' que soy yo, desaparece la posibilidad de lo extraño para los otros. Sin 'lo propio' que son ellos, no me es posible experimentar la interlocución de su extrañeza. Nada es extraño, y por ende tampoco propio, reinando la uniformidad universal de la estructura conjunta que dispone todo como reserva. El engranaje autosuficiente será el futuro de una civilización mundial sin presencia de la diferencia en cualquiera de los ámbitos en que decidamos indagar: la "política" contemporánea y su racionalidad ominosamente estratégica y universalizante, la imposición mundial de una economía de mercado tan culpable como insensible ante la pobreza de un gran porcentaje de la población mundial, la cultura de masas cada vez más invasora y generadora de pautas de acción basadas en arquetipos que corresponden a la presencia global del poder, por ejemplo, ya delinean el triunfo de la unanimidad y la homogeneidad futura del engranaje actual. Parece no haber salida posible en este camino hacia la aniquilación de la diferencia y la pluralidad, no obstante las continuas argumentaciones y detalladas exposiciones de los mismos hijos eruditos del engranaje.

Al indagar por el sentido de 'lo propio', Heidegger no deifica la experiencia familiar de su mundo ni cierra la posibilidad al reconocimiento del 'Otro-extraño'. Va más allá de una apología de 'lo propio' fundada en caracteres metafísicos y excluyentes, que conviertan la diferencia en un absoluto totalitario o en un fundamento universal de lo que es: una defensa ciega del carácter diferencial de lo propio termina negando el valor del Otro y, por esta vía, puede conducir hasta el extremo de propiciar la creación de movimientos de aniquilación de lo extraño. Heidegger, por el contrario, posibilita abogar por el sostenimiento del arraigo como condición de posibilidad de la diferencia en medio de la homogenización técnica de la existencia humana. En el "Festansprache beim Heimatfest in Todtnauberg", y como recuerdo de su experiencia en este pueblo del sur de Alemania, Heidegger afirma: "Cada uno reconoce (anerkennen) al otro en lo que le es propio y lo acepta. Donde siempre y cuando siempre tal cosa acontece a un hombre, será esto un don (Geschenk). Lo que yo en este momento relaté, no es nada especial. Pero es determinante para todo estar-aquí- [existir] con el otro y para el otro (Miteinander und Füreinander-da-sein)" (2000b, p. 644). El vínculo de reconocimiento entre "lo propio' y 'lo Otro' no se reduce, sin embargo, al círculo cercano de lo natal, como una

\footnotetext{
${ }^{41}$ No se trata de 'lo propio' en el sentido de 'lo mío', sino de 'lo propio' como tal en su acepción ontológica. La pregunta por la tierra natal es, en consecuencia, una pregunta por 'lo propio' más allá de 'lo particularmente mío', y de esta manera implica la pregunta por 'lo Otro'. En este contexto, la carencia de tierra natal conlleva a la disolución de lo propio y, con ello, de 'lo Otro', es decir, indiferencia y homogeneidad.
} 
apresurada lectura del texto recién citado puede dar a entender. Este tipo de reconocimiento exclusivo hacia los otros miembros del lugar de origen no sería más que la expresión de un encapsulamiento grupal en lo propio y, con ello, el germen de la exclusión de lo extraño. No es para nada novedoso, entonces, ver en esta actitud hacia el Otro el origen del totalitarismo en cualquiera de sus variadas manifestaciones. Heidegger no postula un fundamento absoluto y comprehensivo de la existencia humana ni concibe la experiencia de 'lo propio' en el sentido restringido de 'aquello que me pertenece'. Es por esta razón que su concepción de la experiencia del propio arraigo no entra en contradicción con el reconocimiento de los 'propios arraigos ajenos', y que la defensa de su tierra natal no pretende negar el modo particular de experiencia de lo propio del mundo del extraño. Su crítica no está dirigida a otros modos de experimentar lo propio de la tierra natal ni a la existencia del extraño, sino, por el contrario, a la falta de arraigo generadora de uniformidad, que pasa por alto la interpelación del Otro en cuanto tal y de esta manera cae en el olvido de las posibilidades del mutuo reconocimiento que, desde mi punto de vista, presupone la preeminencia ontológicamente fundada del extraño. Podemos valorar la importancia mutua que representan 'lo extraño' y 'lo propio', acudiendo a lo sostenido por Heidegger en la alocución festiva de Todtnauberg: "Para las personas nativas de un lugar (Einheimischen) y para los huéspedes es necesario en la misma medida aquel reconocimiento mutuo y aquella aceptación de lo propio que, por otra parte, solamente es posible cuando por doquier el sentido para los 'Sachen ehne dra' ('asuntos o cosas más allá de lo aprehensible') permanece vivo" (2000b, p. $648)^{42}$. Contrario a lo afirmado de manera apresurada en torno a la pregunta heideggeriana por lo propio de la tierra natal, ésta no puede ser entendida como una indagación restrictiva de la diferencia -la marca de su agonía- sino, por el contrario, como una profunda afirmación del carácter constitutivo de ésta en la auténtica (eigene) experiencia humana. La pregunta por el sentido de la tierra natal puede ser interpretada, entonces, como una forma filosóficamente fundada de afirmar la diferencia, que en tanto expresión del arraigo abierto al mundo sigue siendo la manifestación de la existencia humana como posibilidad arrojada.

\section{Referencias bibliográficas}

Grimm, J. y Grimm, W. (1956): Deutsches Wörterbuch (12/I), Leipzig, Hirzel. HeidegGer, M. (2006): Identität und Differenz, Gesamtausgabe 11, Frankfurt am Main, Klostermann.

\footnotetext{
42 Para ver el significado de 'Sachen ehne dra' como 'asuntos o cosas más allá de lo aprehensible' ver 2000b, pp. 646 s.
} 
HeidegGer, M. (2000a): Vorträge und Aufsätze, Gesamtausgabe 7, Frankfurt am Main, Klostermann.

HeidegGer, M. (2000b): Reden und andere Zeugnisse eines Lebensweges, Gesamtausgabe 16, Frankfurt am Main, Klostermann.

HeidegGer, M. (1997): Der Satz vom Grund, Gesamtausgabe 10, Frankfurt am Main, Klostermann.

HeidegGer, M. (1985): Unterwegs zur Sprache, Gesamtausgabe 12, Frankfurt am Main, Klostermann.

HeIdegGer, M. (1984): Hölderlins Hymne $>>$ Der Ister $<<$, Gesamtausgabe 53, Frankfurt am Main, Klostermann.

HeIdegGer, M. (1983a): Die Grundbegriffe der Metaphysik. Welt - Endlichkeit Einsamkeit, Gesamtausgabe 29/30, Frankfurt am Main, Klostermann.

HeidegGer, M. (1983b): Aus der Erfahrung des Denkens, Gesamtausgabe 13, Frankfurt am Main, Klostermann.

HeIdegGer, M. (1982): Hölderlins Hymne $>>$ Andenken $<<$, Gesamtausgabe 52, Frankfurt am Main, Klostermann.

HeidegGer, M. (1980): Erläuterungen zu Hölderlins Dichtung, Gesamtausgabe 4, Frankfurt am Main, Klostermann.

HeidegGer, M. (1977a): Sein und Zeit, Gesamtausgabe 2, Frankfurt am Main, Klostermann.

HeidegGer, M. (1977b): Holzwege, Gesamtausgabe 5, Frankfurt am Main, Klostermann.

HeIDEGGeR, M. (1976): Wegmarken, Gesamtausgabe 9, Frankfurt am Main, Klostermann.

HeidegGer, M. (1959): Gelassenheit, Pfullingen, Neske.

LEvinas, E. (2008): Totalität und Unendlichkeit, Freiburg/München, Alber, 2008

Marten, R. (1980): "Heideggers Heimat. Eine philosophische Herausforderung", en: U. Guzzoni: Nachdenken über Heidegger. Eine Bestandsaufnahme, Hildesheim, Gerstenberg, pp 136-159.

Welsch, W. (1985): "Zwischen Universalismus und Partikularismus. Zum Verhältnis von Philosophie und Heimat in Antike, Neuzeit und Postmoderne unter besonderer Berücksichtigung Heideggers", en: Stadt Messkirch (ed.): Heimat der Philosophie, Messkirch, Drückerei Heinz Schönbeck, pp. 83-111.

\footnotetext{
Alfredo Rocha de la Torre

Gastwissenschaftler Alexander von Humboldt-Stiftung

Bergische Universität Wuppertal

rochtorre@yahoo.com
} 